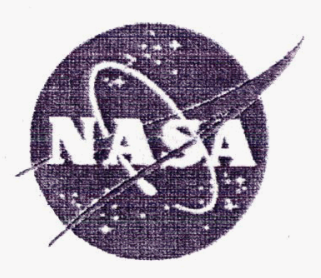

From the Big Bang to Black Holes

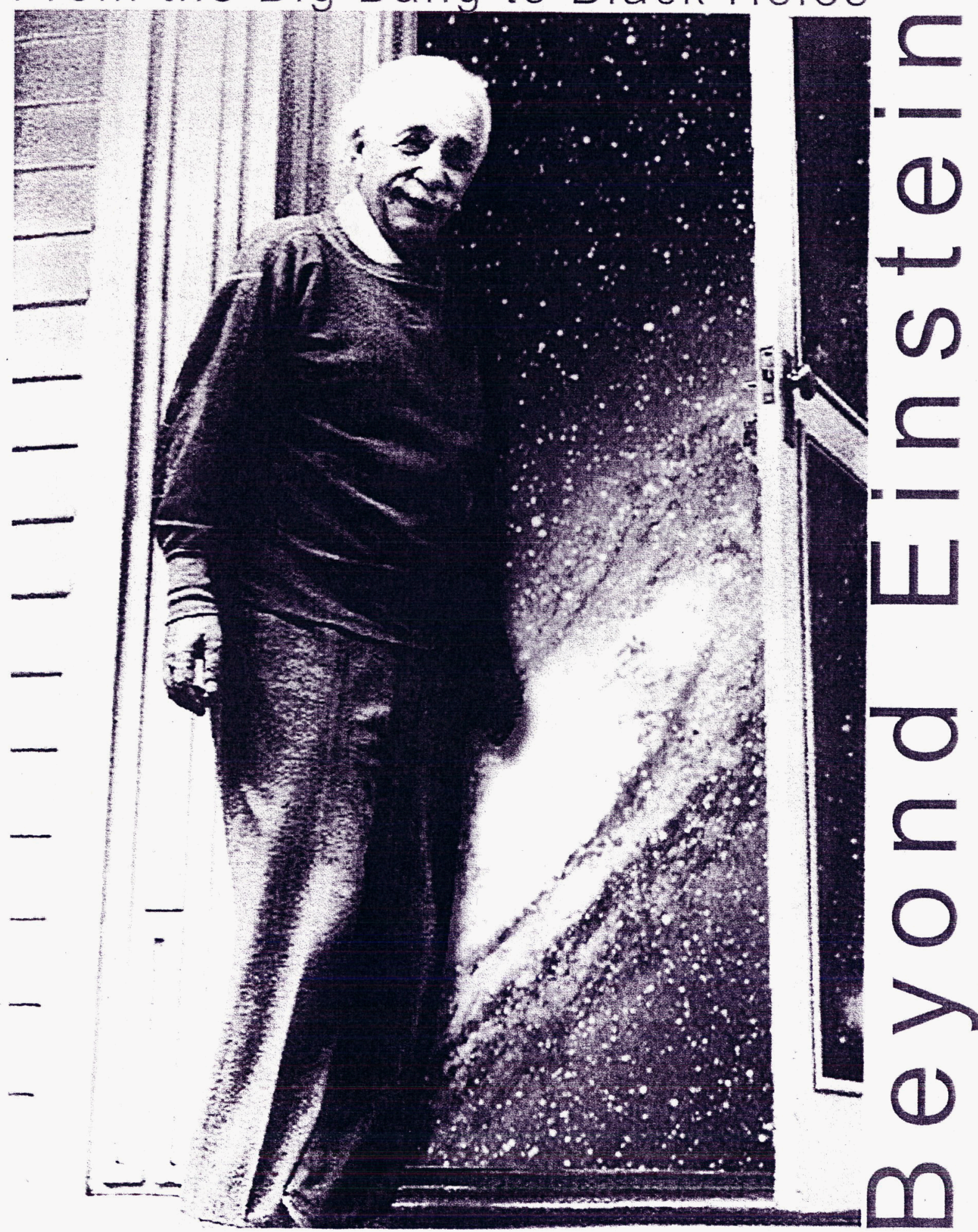

September 2005 
The discoveries of Albert Einstein sparked the scientific revolution of the 20th century and rank among humanity's greatest achievements. Recent developments show that we can now complete Einstein's legacy and, in the first decades of the 21 st century, unravel the mysteries of the Universe that await us . . .

Second Printing: July 2005

Einstein images are used by permission of Roger Richman Agency representing Hebrew University and the Albert Einstein estate. Image of Einstein riding bicycle courtesy of the Archives, California Institute of Technology.

Photo of Richard Feynman by Floyd Clark/Courtesy of Caltech Archives.

Einstein letter to Georges Lemaitre. Permission to quote granted by the Albert Einstein Archives, the Hebrew University of Jerusalem, as well as by the Einstein Papers Project. 


\section{Beyond Einstein}

How did the Universe begin? Does time have a beginning and an end? Does space have edges? The questions are clear and simple. They are as old as human curiosity. But the answers have always seemed beyond the reach of science. Until now.

In their attempts to understand how space, time, and matter are connected, Einstein and his successors made three predictions. First, space is expanding from a Big Bang; second, space and time can tie themselves into contorted knots called "black holes" where time actually comes to a halt; third, space itself contains some kind of energy that is pulling the Universe apart. Each of these three predictions seemed so fantastic when it was made that everyone, including Einstein himself, regarded them as unlikely. Incredibly, all three have turned out to be true. Yet Einstein's legacy is one of deep mystery, because his theories are silent on three questions raised by his fantastic predictions: (1) What powered the Big Bang? (2) What happens to space, time, and matter at the edge of a black hole? (3) What is the mysterious dark energy pulling the Universe apart?

The answers to these questions - which lie at the crux of where our current theories fail us-will lead to a profound, new understanding of the nature of time and space. To find answers, however, we must venture beyond Einstein. The answers require new theories, such as the inflationary Universe and new insights in high-energy particle theory. Like Einstein's theories, these make fantastic predictions that seem hard to believe: unseen dimensions and entire universes beyond our own. We must find facts to confront and guide these new theories. Powerful new technologies now make this possible. And NASA and its partners are developing an armada of space-based observatories to chart the path to discovery.

Here is where the Beyond Einstein story begins. By exploring the three questions that are Einstein's legacy, we begin the next revolution in understanding our Universe. We plot our way forward using clues from observations and from new ideas connecting the worlds of the very small and the very large, from the atom out through the deepest reaches of the cosmos.

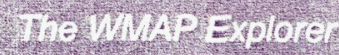

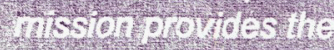

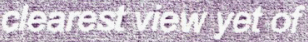

He peryus morowans

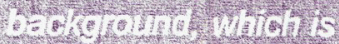

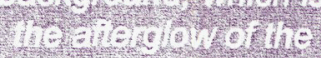

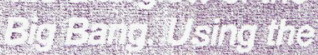

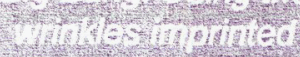
$27.06010212=$

\section{MAPping the Big Bang Afterglow}

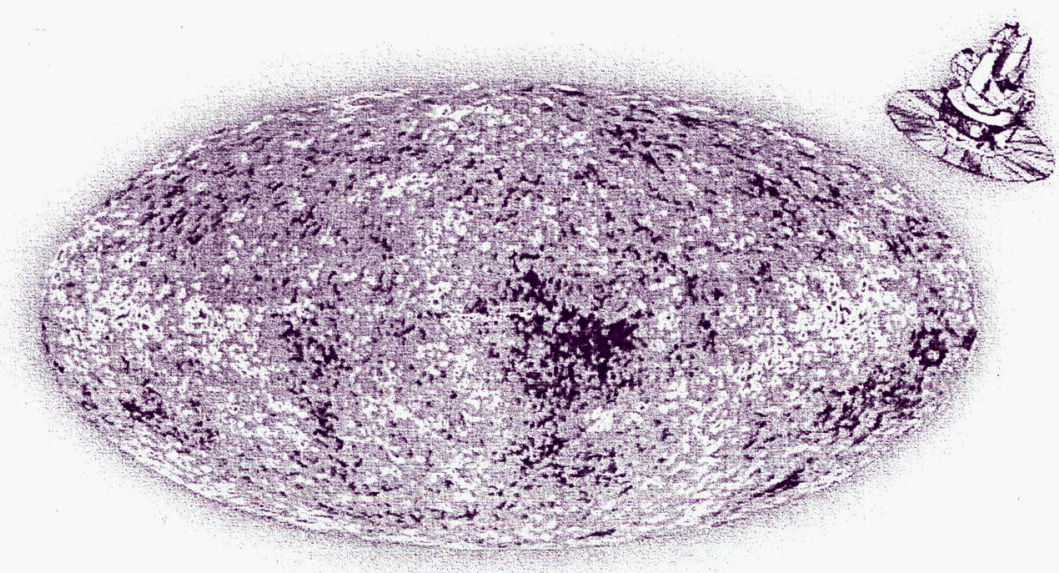

The map of the cosmic microwave background, the afterglow of the Big Bang, obtained by NASA's Wilkinson Microwave Anisotropy Probe (WMAP). 


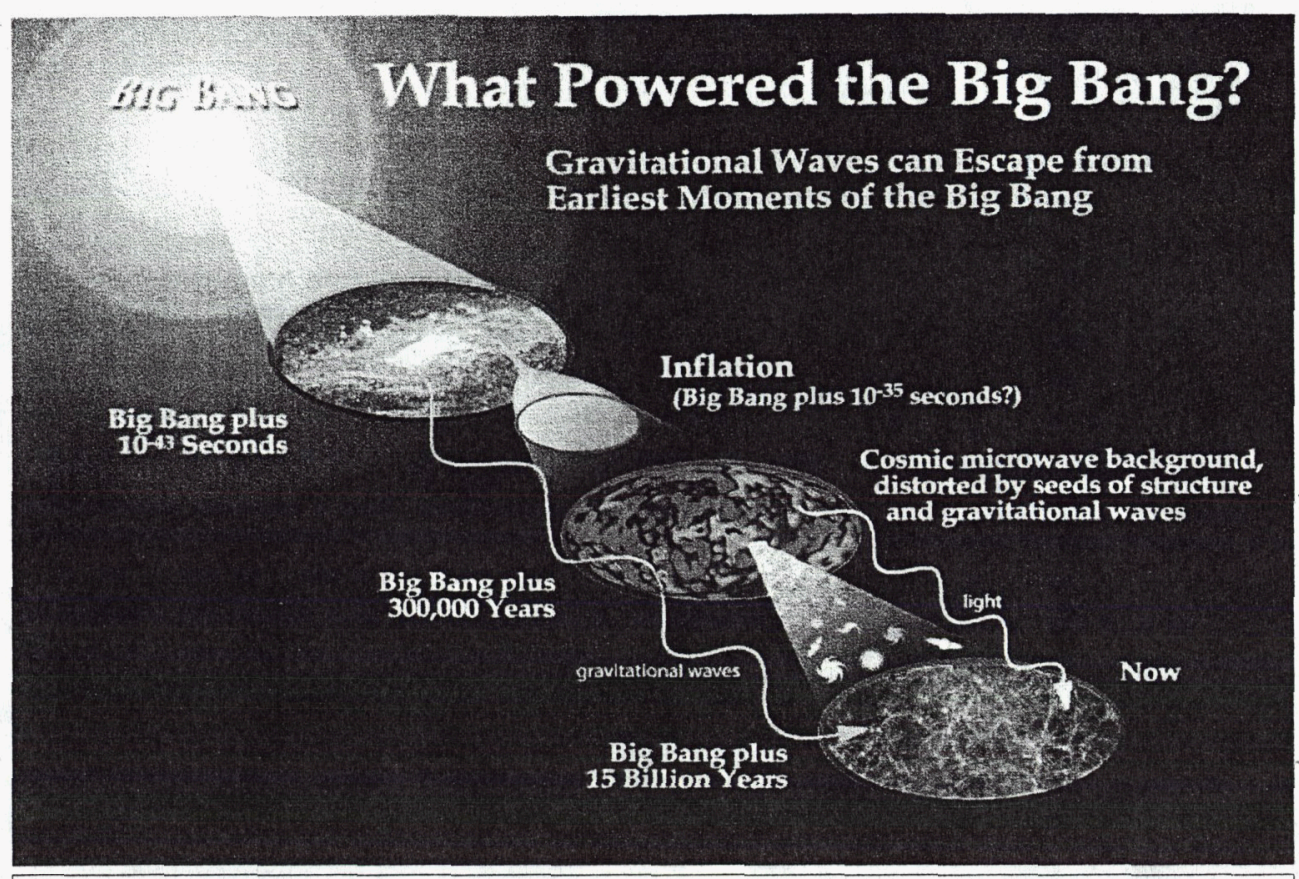

The answer to what powered the Big Bang is imprinted in its afterglow, called the cosmic microwave background, which is still visible for us to study today.

\section{What powered the Big Bang?}

If the Universe is expanding, as countless observations have shown, it is only logical to assume that the Universe was more compact yesterday, even more compact the day before, and so on. At some point in the distant past, all the matter and energy we see today must have been confined to a microscopic region of unimaginably high density. This would be the moment of the Big Bang.

We know a great deal about the history of the Universe, from its hot, formless beginnings to the glorious hierarchal structure of stars and galaxies in the modern era. Clues to the past are all around us. In the 1960s scientists identified a pervasive microwave radiation emanating from all points in the sky. This radiation, called the cosmic microwave background, is the afterglow of the Big Bang itself. Embedded in this afterglow are slight temperature fluctuations - a little warmer here, a little cooler there- that point back to slight density differences in the infant Universe - a little more matter here, a little less there. These fluctuations are the seeds of all the structure we see today.

NASA's Cosmic Background Explorer (COBE) satellite discovered the fluctuations and, most recently, NASA's Wilkinson Microwave Anisotropy Probe (WMAP) has refined the measurement. We see how gravity has pulled these wrinkles into stars and planets. We can even determine the ratio the ratio of matter to energy, the era of first starlight, and the age of the Universe, 13.8 billion years.

Most of the X-ray sources seen in this 12-day exposure by the Chandra $\mathrm{X}$-ray Observatory are active galaxies and quasars powered by massive black holes. Ground-based observations show that many of them are shrouded by dust; many others remain unidentified, invisible except in $\mathrm{X}$ rays.

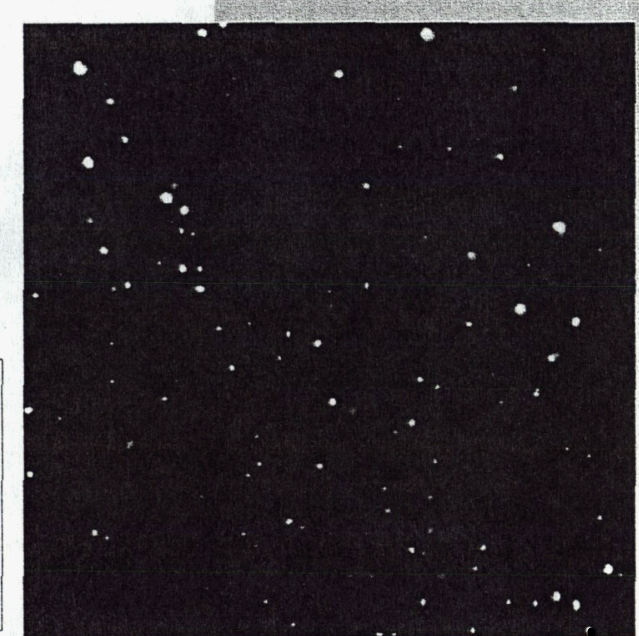




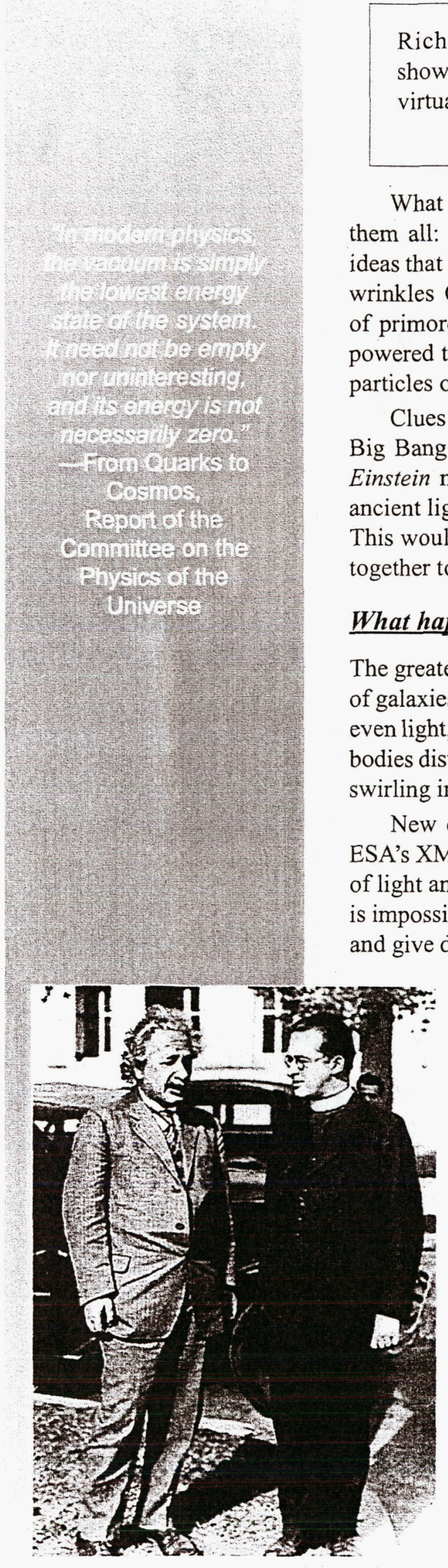

Richard Feynman [Nobel Prize, 1965] showed that "empty" space was filled with virtual particles.

What we don't know is the most basic fact of hem all: What started it all? Modern theoretical ideas that try to answer this question predict that the wrinkles COBE discovered arose from two kinds of primordial particles: from the energy field that powered the Big Bang; and gravitons, fundamental rticles of space and time.

Clues to the nature of these particles exist in the Big Bang afterglow. Measurements from Beyond Einstein missions will coax information from this
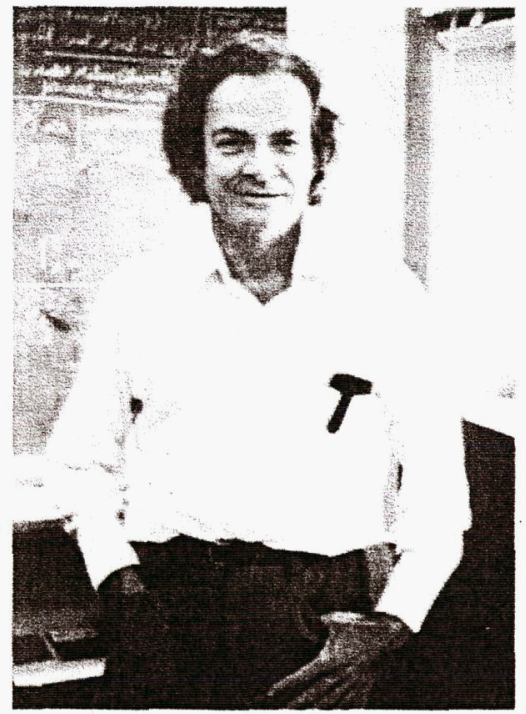
ingt, which has held its secrets for so long. This would enable us to piece together the story of how time, space, and energy worked of galaxies and by the collapse of stars. Gravity is so overpowering here that nothing, not even light, can escape its grasp. By definition black holes are invisible. Yet these invisible bodies disturb space considerably, offering us two ways to study them: by observing matter wirling into them, and by listening to the waves of distortion they make in spacetime.

New data from X-ray satellites, such as NASA's Chandra X-ray Observatory and ESA's XMM-Newton, show signs of gas whizzing about black holes at close to the speed of light and hint that time is slowing as the gas plunges into the zone from which escape is impossible. Beyond Einstein missions will take a census of black holes in the Universe and give detailed pictures of what happens to space and time at the edges of these roiling vortices.

One key mission will create movies from the X-ray light emitted from multimillion-degree gas as it approaches a black hole's border, called the event horizon. Another mission will listen for gravitational waves, which are ripples in spacetime predicted by Einstein. These waves are created by black hole mergers; they move across the "sea" of space at light speed, unperturbed, and offer an unobstructed view of these powerful collisions.

Einstein himself never dreamed that it would be possible to detect gravitational waves, which only distort the distance between objects as far apart as the Earth and Moon by less than the width of an atom. Yet the technology now exists to do so.

"I found it very ugly indeed that the field law of gravitation should be composed of two logically independent terms which are connected by addition. About the justification of such feelings concerning logical simplicity it is difficult to argue. I cannot help to feel it strongly and I am unable to believe that such an ugly thing should be realized in nature." -Albert Einstein, in a Sept. 26, 1947, letter to Georges Lemaître 
The landmark discovery of the 1990s was that the expansion of the Universe is accelerating. The greatest mystery in astronomy today is the nature of this force opposing gravity, which we call "dark energy."

Because Einstein originally thought the Universe was static, he conjectured that even the emptiest possible space, devoid of matter and radiation, might still have an energy countering gravity and keeping the Universe from collapsing. He called it a "Cosmological Constant." When Edwin Hubble discovered the expansion of the Universe, Einstein rejected his own idea, calling it his greatest blunder.

But the Universe isn't just expanding; the expansion rate, which appears to have slowed several billion years ago, is revving up. We live in a runaway Universe, in which the most distant galaxies visible today will soon fly off forever beyond the horizon. This acceleration could be due to the concept that "empty space" isn't empty. Richard Feynman and others who developed the quantum theory of matter realized that empty space is filled with "virtual" particles continually forming and destroying themselves. These particles create negative pressure, like a vacuum energy, that pulls space outward. No one, however, could predict this energy's magnitude.

Independent measurements reveal that dark energy comprises about $70 \%$ of the total mass-energy budget of the Universe. We still do not know whether or how the highly accelerated expansion in the early Universe, called inflation, and the current accelerated expansion, due to dark energy, are related. A Beyond Einstein mission will measure the expansion accurately enough to learn whether this energy is a constant property of empty space, as Einstein conjectured, or whether its strength varies over time, a property predicted by modern theories of the forces of nature.

\section{The Beyond Einstein Program}

The Beyond Einstein program has three linked elements which advance science and technology towards two visions: to detect gravitational wave signals from the earliest possible moments of the Big Bang, and to image the event horizon of a black hole. The central element is a pair of Einstein Great Observatories: Constellation-X and LISA. These powerful facilities will blaze new paths to the questions about black holes, the Big Bang, and dark energy. They will also address other central goals of contemporary astrophysics. The second element is a series of competitively selected Einstein Probes, each focused on one of the science questions. The third element is a program of technology development, theoretical studies and education to support the Probes and the vision missions: the Big Bang Observer and the Black Hole Imager. The program offers competitive opportunities for mission leadership, technology development, and groundbreaking scientific research, with goals that excite the public.

\section{Einstein Great Observatories}

While these missions are focused on specific observational goals, the capabilities they provide are so dramatically innovative that they will also provide a broad science return that will impact all areas of astrophysics, as have Hubble Space Telescope and the Chandra X-ray Observatory before them.

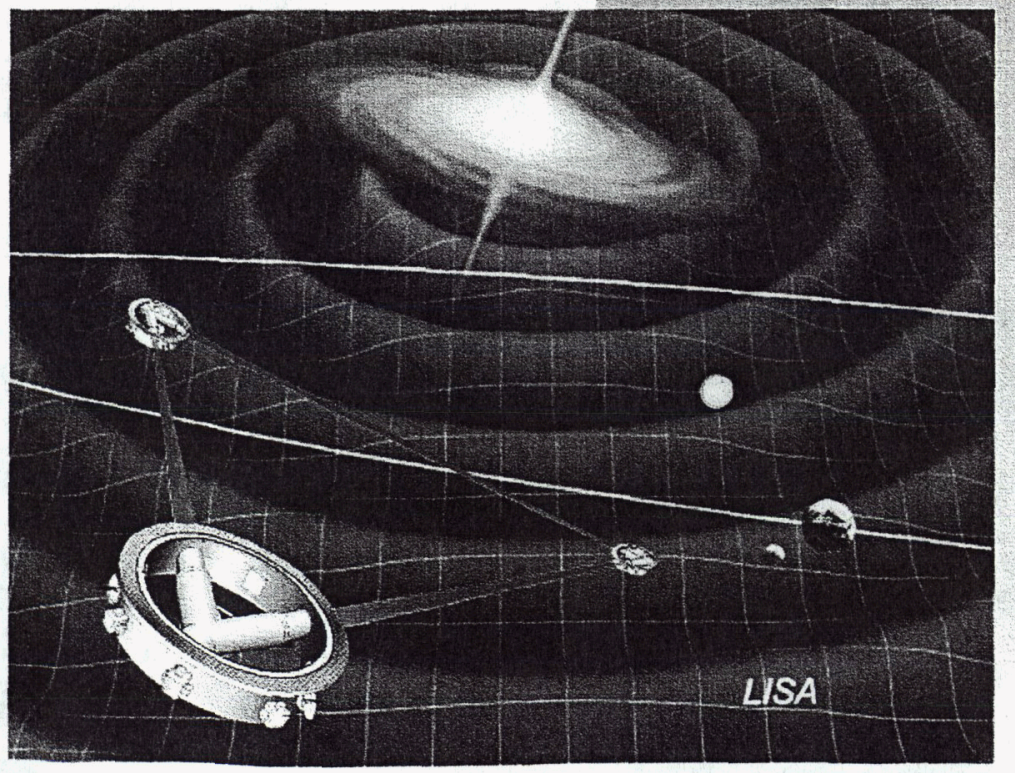


The Laser Interferometer Space Antenna (LISA) will detect gravitational waves. These waves, predicted by Einstein but never detected directly, move at light speed and come in a range of frequencies, just like light waves. LISA's three spacecraft will act like buoys in space to monitor for a passing wave. The spacecraft will be separated from each other by five million kilometers and "tethered" by a laser.

Because gravitational waves hardly interact with matter, they can penetrate the dusty environments surrounding black holes and provide us with an unobstructed view. LISA will probe space and time at the edges of black holes by listening to the sounds of vibrating spacetime: the booming roar of merging supermassive black holes, the chorus of death cries from stars falling toward black holes, and the zipping sound of a newly formed singularity. LISA may even hear whispers from the time of the Big Bang when our three-dimensional space formed within the unseen space of six or seven dimensions, as some new theories predict. LISA will plot the orbits of stars around black holes to test Einstein's theory under extreme conditions.

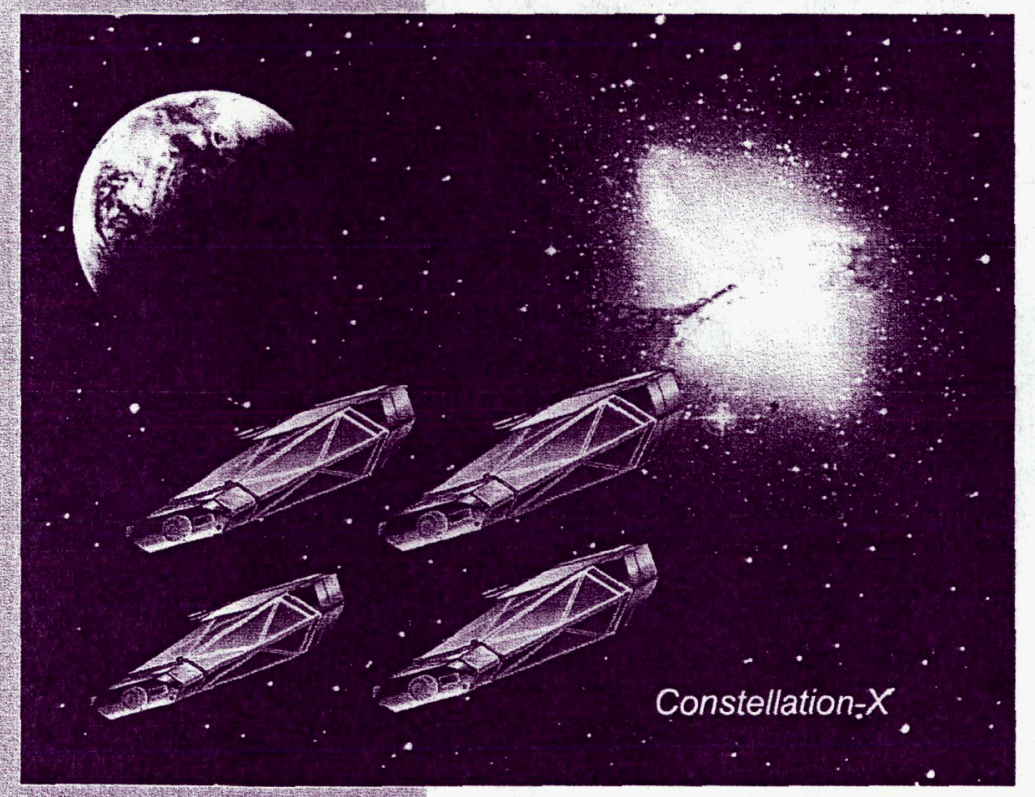

LISA's precise measurements of distances to merging black holes will provide a new yardstick with which to measure the Universe and constrain the nature of dark energy. LISA will also detect gravitational waves from thousands of binary star systems, yielding new insights into the formation and evolution of binary stars.

The Constellation-X mission will consist of four X-ray telescopes orbiting and observing in unison. This telescope teamwork will provide nearly 100 times the sensitivity of the pioneering $\mathrm{X}$-ray observatories now in orbit: NASA's Chandra $\mathrm{X}$-ray Observatory, Europe's XMM-Newton and Japan's Suzaku.

The great sensitivity of Constellation- $\mathrm{X}$ will allow us to make "slow-motion movies" of hot gas falling toward a black hole. This is done not through imagery but through a technique called spectroscopy, which is analogous to fingerprinting the gas to determine unique properties, such as temperature, density, velocity and atomic number. We will be able to see how time, from our perspective, comes to a standstill as it approaches the black hole event horizon. Einstein's general relativity makes specific predictions about the behavior of matter near a black hole; and any deviation from theory revealed by Constellation- $\mathrm{X}$ observations will expose flaws in Einstein's math.

Constellation-X will also dramatically increase our ability to obtain high-resolution spectroscopy of faint sources. This will enable us to constrain the nature of dark matter and dark energy by observing their effects on the formation of clusters of galaxies, which are massive yet faint and distant structures. These measurements and those by LISA, the Dark Energy Probe and the Inflation Probe will each constrain different possible properties of dark energy. Together they will lead to a better understanding of dark energy. Similarly, Constellation-X and LISA together will help us understand the origin and nature of the giant black holes in the centers of galaxies, as they grow by consuming gas and whole stars or by merging.

\section{Einstein Probes}

Complementing the facility-class Einstein Great Observatories are three sharply focused missions addressing a specific science question. Answers to these three questions can take us beyond Einstein. 
Many NASA missions have laid the groundwork for the Beyond Einstein program and will complement it. NASA's $C O B E$ discovered the first evidence for primordial density fluctuations in the Cosmic Microwave Background. NASA's balloon program (e.g., the NASA/NSF/Italian/UK BOOMERanG) has supported the discovery of the interaction of those fluctuations with matter in the Universe. NASA's WMAP satellite and the ESA's planned Planck satellite will extend these discoveries and are vital precursors to the proposed Inflation Probe.

The Hubble Space Telescope has helped to find and measure the distant supernovae that have forced us to accept the reality of dark energy.

X-ray missions, including NASA's Chandra X-ray Observatory and RXTE, ESA's $X M M$-Newton, and Japan's $A S C A$, have discovered X rays from matter spiraling into black holes, illustrating the potential of Constellation-X.

$G P-B$ will test one of Einstein's exotic predictions: that the rotation of the Earth drags space and time around the Earth into a mild version of the tremendous vortical spin near a spinning black hole.

Swift studies gamma-ray bursts, believed to be a result of the stellar explosions and mergers which create black holes. Swift also tests technology for the Black Hole Finder Probe.

Japan/NASA's Suzaku, launched in July 2005, will be the first to use NASAprovided micro-calorimeters that are prototypes for Constellation-X detectors.

GLAST will provide more sensitivity and energy coverage than ever before for the study of high-energy emissions from particles accelerated in gamma-ray bursts and in the jets from spinning black holes.

ST-7 and ESA's SMART-2 will provide a flight comparison of two disturbance reduction technologies competing for use on LISA.

"How did the Universe begin?" The Universe appears to have begun with a period of inflation, when it expanded faster than the speed of light. The rapid expansion enabled slight density differences in that incredibly dense kernel to ultimately grow into the stars, galaxies and vast voids we see today. Yet what propelled this inflation? Is it the same force that is accelerating the expansion of the Universe today? The Einstein Inflation Probe will investigate.

Inflation theory predicts that this expansion was driven by a quantum fluctuation, a split-second change in energy that created negative pressure, or a vacuum energy, which pulled the Universe apart. The Inflation Probe will look for relics of quantum fluctuations hidden, like fossils, in the afterglow of the Big Bang. This afterglow is a pervasive blanket of microwave radiation, called the cosmic microwave background, still visible today across the entire sky.

The gravitational waves from the Big Bang should have created an imprint on the cosmic microwave background-a unique pattern of polarization that speaks of these quantum fluctuations. One way to examine the theorize inflationary period is to study cosmic microwave background polarization, similar to how WMAP is measuring temperature fluctuations today.

"How do black holes form and grow?" Scientists have identified two main classes of black holes: smaller, stellar-sized black holes that form from the collapse of massive stars; and supermassive black holes in the core of most galaxies. The latter can contain the mass of millions to billions of suns and grow by swallowing stars and gas that venture

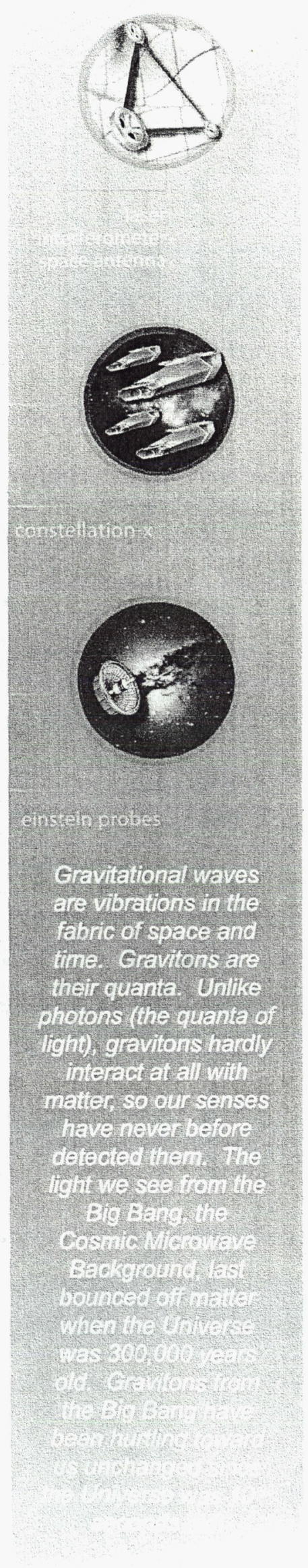




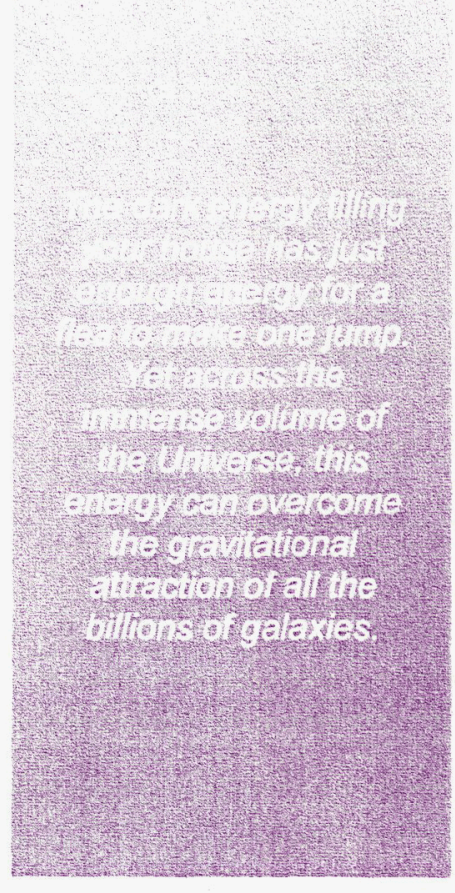

too close. This consumption releases great amounts of energy. But there is an accounting problem: Not enough light is coming from supermassive black holes to explain their growth. There are hints that much of the growth occurs behind a shroud of dust. The "Black Hole Finder Probe" will conduct a census of hidden black holes, revealing where, when, and how they form. This probe will also identify new sources for the powerful Constellation $\mathrm{X}$-ray Observatory to study in detail.

"Why is the Universe accelerating?" This is a question that would not have been asked five years ago, before there was evidence that the Universe was being pulled apart. To understand this energy, we must measure the expansion of the Universe with high precision. This will require the most precise cosmic yardsticks we can find. Several ideas for such a joint dark energy mission with the Department of Energy have been proposed-for example, precision measurement of distant supernovae by a wide-field space telescope. Independent diagnostics of the dark energy will be crucial to verify the validity of results and to increase the precision of the measurements. The Inflation Probe and LISA will provide completely independent cosmic yardsticks with which to measure the effects of dark energy, and Constellation- $X$ will observe the first clusters of galaxies whose evolution depends critically on dark matter and dark energy.

\section{The Beyond Einstein Program}
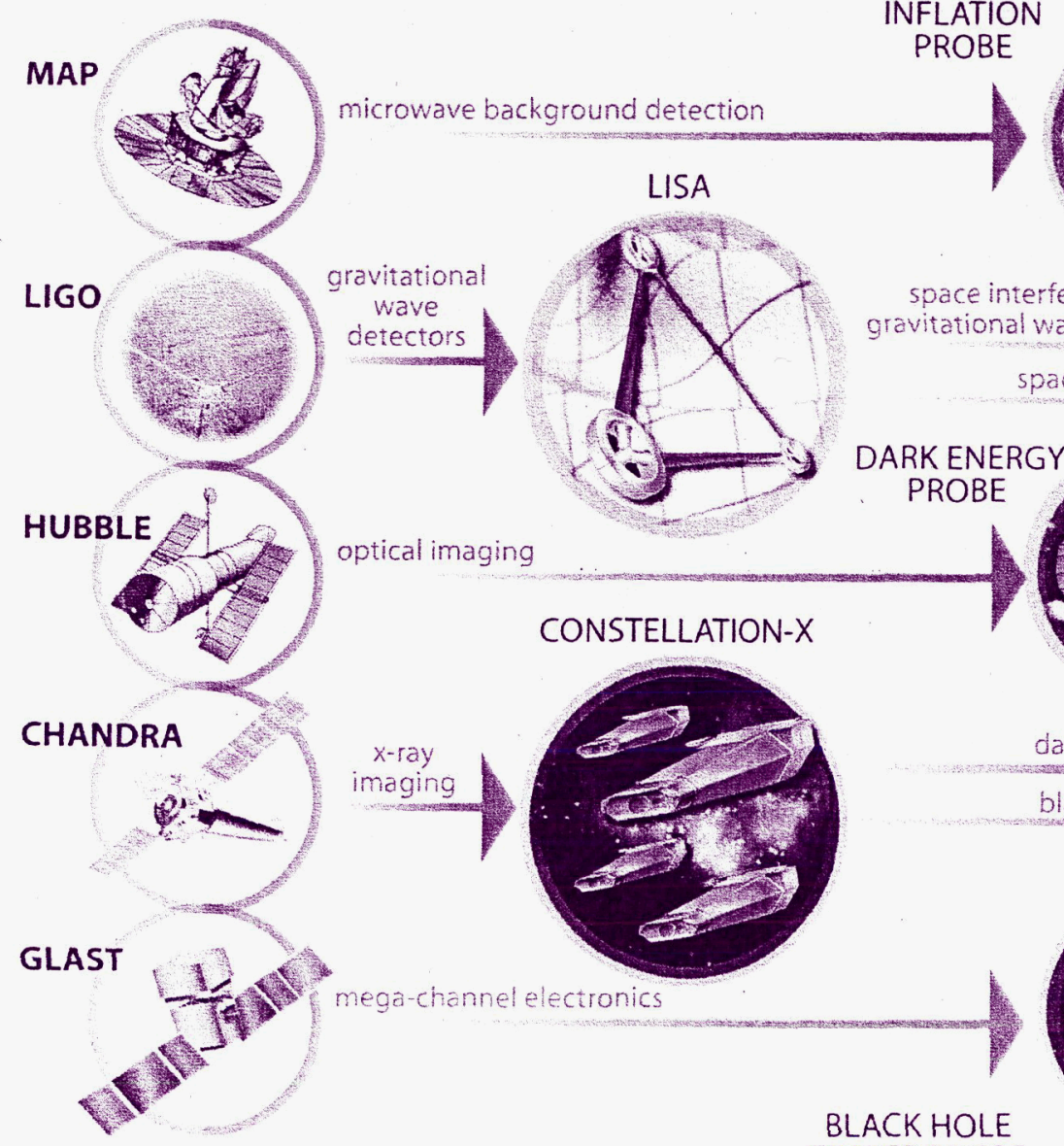

INFLATION

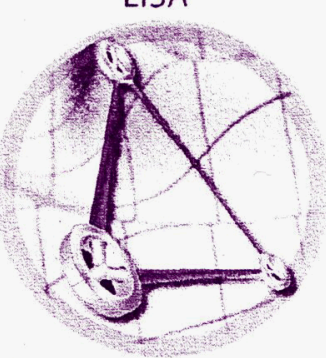

space interferometry, gravitational wave detection
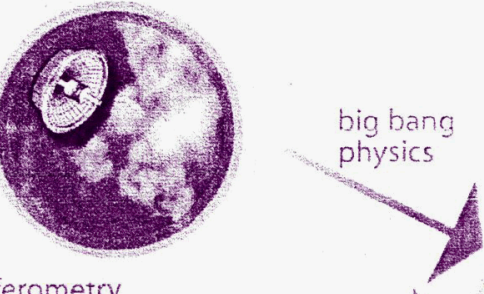
BIG BANG OBSERVER

\section{space interferometry}

DARK ENERGY PROBE
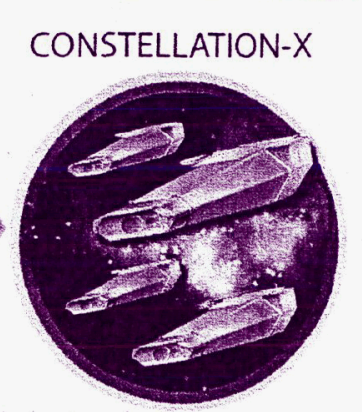

BLACK HOLE

FINDER PROBE 
The Gamma-Ray Large Area Space Telescope (GLAST) is an international and multi-agency (NASA and DOE) project to study the most energetic objects and phenomena in the Universe, such as antimatter and high-power jets of matter shooting away from black holes. GLAST combines the fields of astronomy and high-energy particle physics.

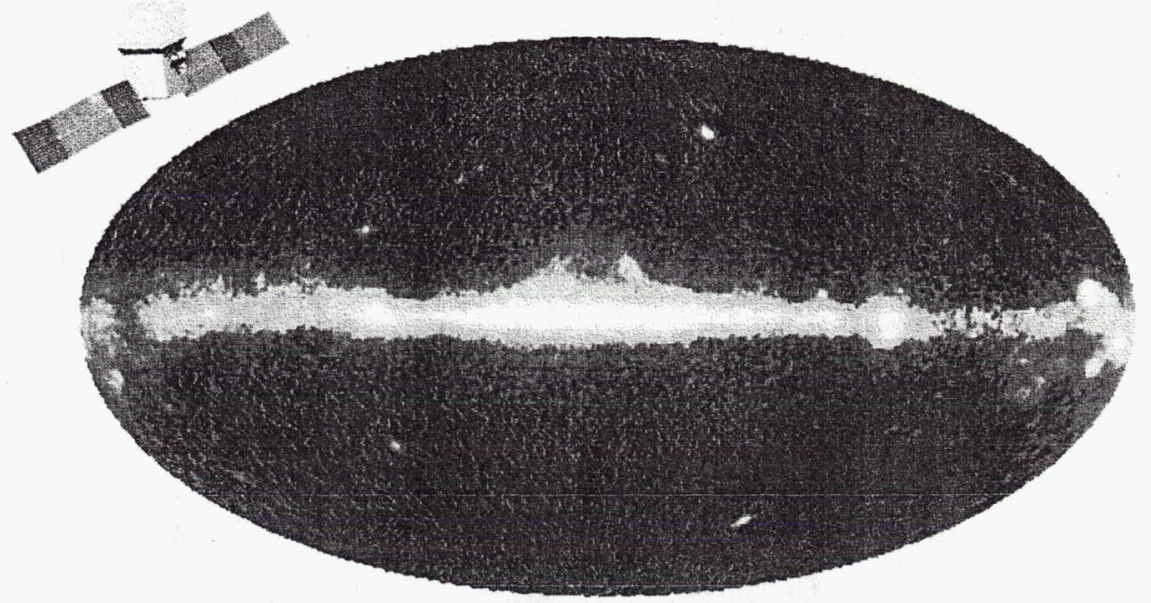

Now under construction, GLAST will build upon the success of the EGRET instrument on the Compton Gamma Ray Observatory. Nearly half of the 271 objects detected by EGRET in the 1990s remain unidentified. GLAST, with more than 30 times the sensitivity of EGRET, is primed for discovery. What could these objects be? Perhaps long-hidden black holes or something new entirely? GLAST may provide the Beyond Einstein missions with vital information about black holes, dark matter, dark energy and gravity.

\section{The Ultimate Vision}

The technology to go far beyond Einstein is within our reach if we approach the grand goals systematically, mission building upon mission, proving and refining the required technology. Strategic investments in hardware, software, and astrophysical theory will lead the way forward to two visions:

To explore the beginning of time, a "Big Bang Observer" will build upon LISA to directly measure gravitons from the early Universe still present today. In contrast to the Inflation Probe's measurement of frozen imprints of much longer waves on the microwave background, the Big Bang Observer will observe gravitational waves in their original form, from still earlier in the Big Bang. The Big Bang Observer would give us a direct view of the creation of spacetime, a truly profound achievement.

To explore the end of time and the edges of space, Constellation-X will measure the spectral signatures of gas swirling into black holes, and LISA will record the tune to which stars dance around it. But there is no substitute for a direct image. A "Black Hole Imager" based on X-ray interferometry, could take this epochal picture, revealing directly the fate of matter near a black hole. 


\section{$\underline{\text { Technology }}$}

While the enabling foundations are well in hand, the Beyond Einstein program demands many refinements in technology. Constellation-X will need lightweight optics and cryogenic X-ray calorimeters. LISA will need sensitive monitoring units coupled to micro-newton thrusters to keep its test masses free of non-gravitational forces. It will also require very stable laser measurement systems. The Einstein Probes require study of a broad range of technologies so that the most effective approach to their science goals can be chosen. The vision missions, Black Hole Imager and Big Bang Observer, need still greater precision in spacecraft pointing and control.

\section{Research and Analysis}

The R\&A program is the cradle for the necessary technology and for the theoretical underpinnings of NASA space science missions. It is the first step in a process that turns ideas into missions, as well as the final step in turning missions into scientific advances. NASA's R\&A program draws heavily on the resources of our universities, providing an additional benefit: the training of students who become the architects and builders of future missions. The Einstein Probes require new detectors for which ground-based and balloon tests will be essential. Laboratory measurements of atomic data are necessary to link observations to scientific conclusions. Theory provides the intellectual context for any scientific effort. Theoretical work is essential to the conception and design of missions and to the interpretation of the data they provide--especially for the Beyond Einstein missions, which are designed to test predictions that challenge our beliefs.

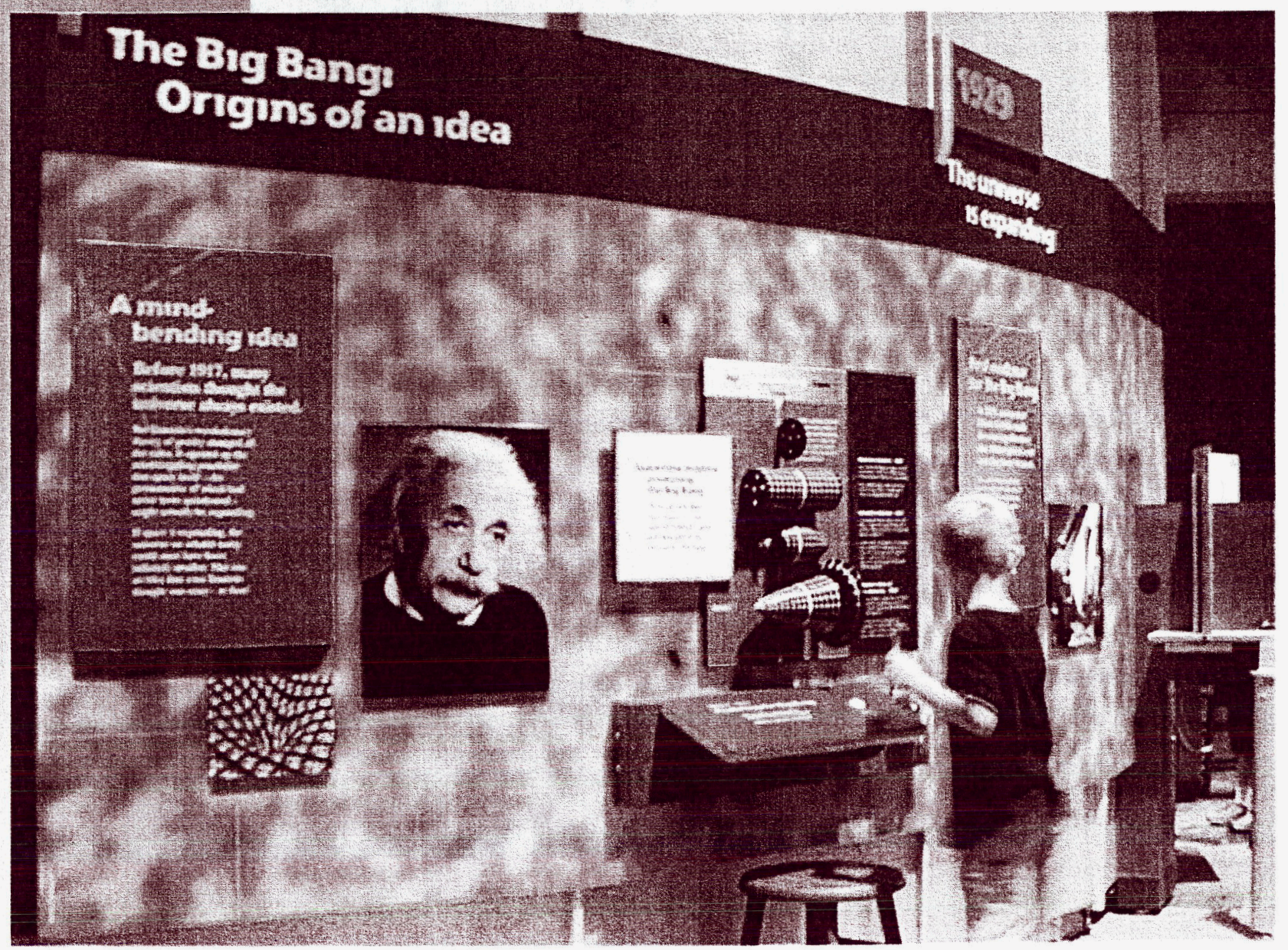




\section{Education and Public Outreach}

Beyond Einstein offers an unparalleled opportunity to involve the public in the excitement of cosmic exploration, and to inspire and cultivate the next generation of scientists and engineers. The public's eagerness to share this adventure is reflected in part by the many popular news reports, bestselling books, television series, and even Hollywood movies that draw on Beyond Einstein themes. The television shows and educational materials for "Live from a Black Hole" and "Live from the Edge of Space" reached an estimated five million students. Public television's NOVA program on dark energy (Runaway Universe) was seen by millions of Americans. The origin of the Universe and black holes are central to K-12 science literacy standards and curricula. The Beyond Einstein themes will soon provide the majority of materials on these subjects in our nation's schools, and the missions will weave an ongoing story that is one of the most compelling in all science.

\section{Einstein's Legacy}

In 1905 an unknown patent clerk in Bern, Switzerland, named Albert Einstein ignited a scientific revolution. Practicing physics on the sly and with very little personal interaction with the scientific establishment at the time, Einstein wrote five seminal papers that year that encompassed the whole of the universe, from the workings of the atom and out through space and time. One hundred years onward, still we marvel. And we struggle to make sense of Einstein's outrageous legacy - spacetime warps, an accelerating universe, black holes, possible hidden dimensions, and the notion that all of space is quantized, built from infinitesimal, discrete bundles of matter and energy.

Einstein sought, but never achieved, an understanding of how nature works at its deepest level. We now seek to fulfill Einstein's quest through a program of missions we are designing today to carry out over the next decade.

There is tangible excitement among scientists. We are on the verge of the next scientific breakthrough that will define the 21 st century and beyond. This is the new scientific revolution, a race for the next great leap forward. Scientists around the world from a variety of once-disparate disciplines are uniting for the cause. Thus, we will follow matter to the very brink of black holes and detect particles of time left over from the beginning of the universe. And we will use breakthrough technologies to see beyond the vision of Einstein-to the uttermost extremities of existence.
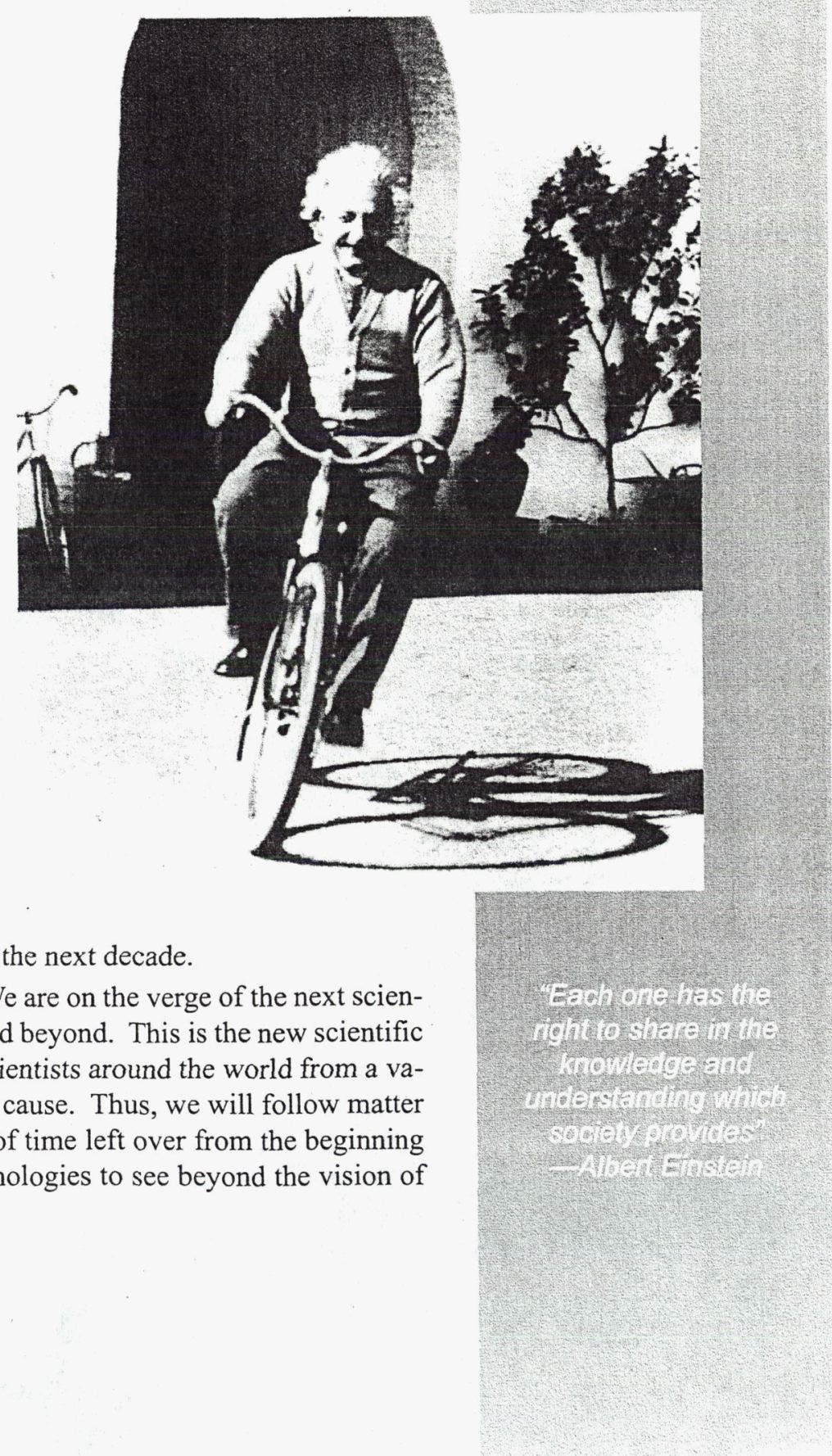


\section{Sources of Further Information}

- NASA Science Mission Directorate

http://science.hq.nasa.gov/

- Beyond Einstein

http://universe.nasa.gov/

- Laser Interferometer Space Antenna (LISA)

http://lisa.nasa.gov/

- Constellation-X

http://constellation.gsfe.nasa.gov/

- Suzaku

http://suzaku.gsfc.nasa.gov/docs/astroe/astroegof.html

- Swift

http://swift. gsfc.nasa.gov/

- Chandra X-ray Observatory

http://chandra.harvard.cdu/

- Wilkinson Microwave Anisotropy Probe (WMAP) http://map.gsfc.nasa.gov/

- Space Technology 7 http://nmp.jpl.nasa.gov/st7/

- High Energy Physics http://doe-hep.hep.net/ http:/doe-hep.hep.net/HEPAP/Irp_report0102.pdf

- Laboratory Astrophysics http:/web99.arc.nasa.gov/ astrochem/nasalaw/whitepaper.html

- Rossi X-ray Timing Explorer (RXTE) http:/heasarc.gsfe.nasa.gov/docs/xte/xte_1st.html

- Gamma-ray Large Area Space Telescope (GLAST) http:/glast.gsfc.nasa.gov

- Gravity-Probe B http:/einstein.stanford.cdu

- Planck

http:/sci.esa.int/home/planck 


\section{Beyond Einstein Timeline}

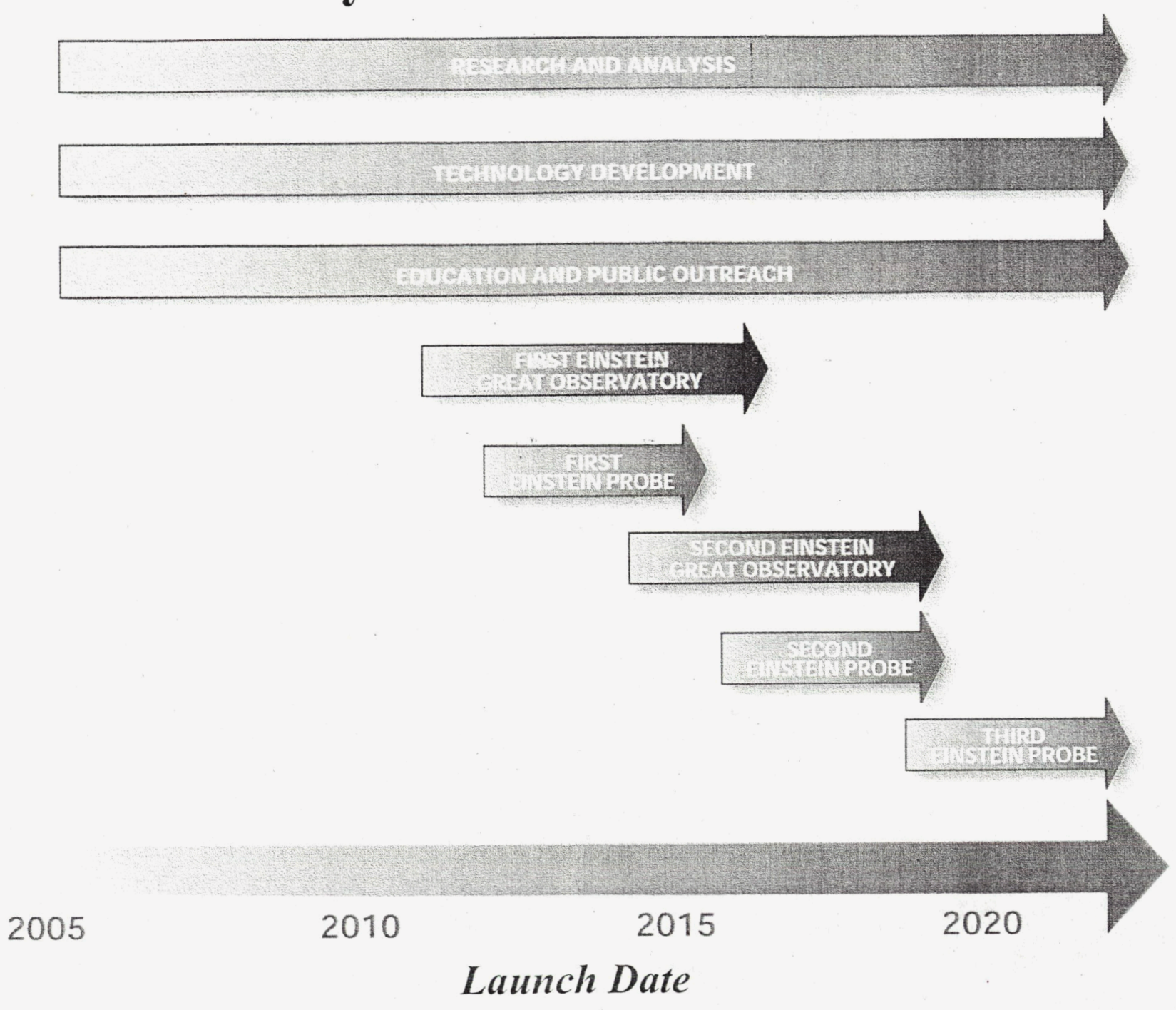




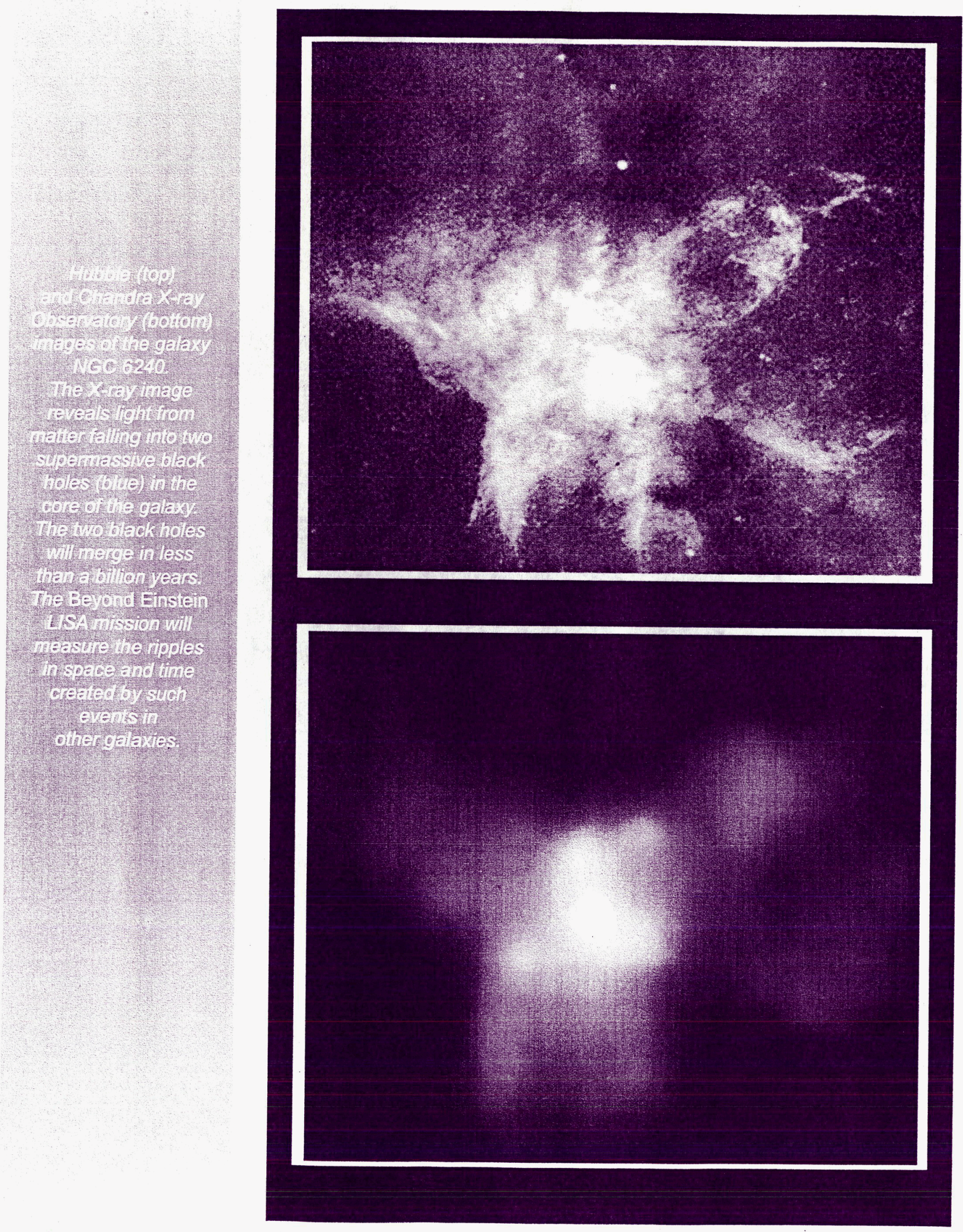




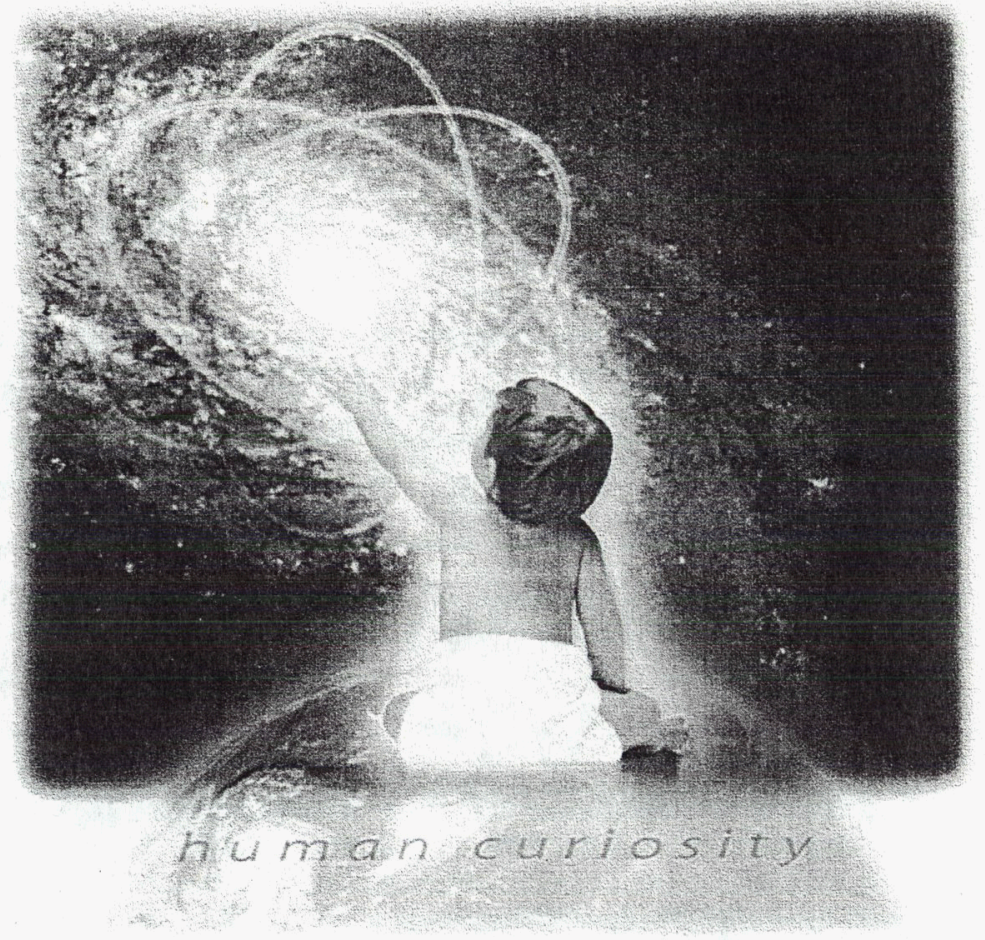

NASA: Explore. Discover. Understand.

http://www.nasa.gov/ 